\title{
Reproductive aging-associated common genetic variants and the risk of breast cancer
}

\author{
Chunyan He ${ }^{1,2^{*}}$, Daniel I Chasman ${ }^{3}$, Jill Dreyfus ${ }^{4}$, Shih-Jen Hwang ${ }^{5}$, Rikje Ruiter ${ }^{6}$, Serena Sanna ${ }^{7}$, Julie E Buring ${ }^{3}$, \\ Lindsay Fernández-Rhodes ${ }^{8}$, Nora Franceschini ${ }^{8}$, Susan E Hankinson ${ }^{9,10}$, Albert Hofman ${ }^{6}$, Kathryn L Lunetta ${ }^{5,11}$, \\ Giuseppe Palmieri ${ }^{12}$, Eleonora Porcu ${ }^{7}$, Fernando Rivadeneira ${ }^{6,13,14}$, Lynda M Rose ${ }^{3}$, Greta L Splansky ${ }^{5}$, \\ Lisette Stolk ${ }^{13,14}$, André G Uitterlinden ${ }^{6,13,14}$, Stephen J Chanock ${ }^{15}$, Laura Crisponi ${ }^{7}$, Ellen W Demerath ${ }^{4}$, \\ Joanne M Murabito ${ }^{5,16}$, Paul M Ridker ${ }^{3}$, Bruno H Stricker ${ }^{6,13}$ and David J Hunter ${ }^{9,10,17}$
}

\begin{abstract}
Introduction: A younger age at menarche and an older age at menopause are well established risk factors for breast cancer. Recent genome-wide association studies have identified several novel genetic loci associated with these two traits. However, the association between these loci and breast cancer risk is unknown.

Methods: In this study, we investigated 19 and 17 newly identified single nucleotide polymorphisms (SNPs) from the ReproGen Consortium that have been associated with age at menarche and age at natural menopause, respectively, and assessed their associations with breast cancer risk in 6 population-based studies among up to 3,683 breast cancer cases and 34,174 controls in white women of European ancestry. In addition, we used these SNPs to calculate genetic risk scores (GRSs) based on their associations with each trait.

Results: After adjusting for age and potential population stratification, two age at menarche associated SNPS (rs1079866 and rs7821178) and one age at natural menopause associated SNP (rs2517388) were associated with breast cancer risk ( $p$ values, $0.003,0.009$ and 0.023 , respectively). The odds ratios for breast cancer corresponding to per-risk-allele were 1.14 ( $95 \% \mathrm{Cl}, 1.05$ to 1.24$), 1.08$ (95\% Cl, 1.02 to 1.15$)$ and 1.10 (95\% Cl, 1.01 to 1.20$)$, respectively, and were in the direction predicted by their associations with age at menarche or age at natural menopause. These associations did not appear to be attenuated by further controlling for self-reported age at menarche, age at natural menopause, or known breast cancer susceptibility loci. Although we did not observe a statistically significant association between any GRS for reproductive aging and breast cancer risk, the $4^{\text {th }}$ and $5^{\text {th }}$ highest quintiles of the younger age at menarche GRS had odds ratios of 1.14 (95\% Cl, 1.01 to 1.28) and 1.13 (95\% $\mathrm{Cl}, 1.00$ to 1.27), respectively, compared to the lowest quintile.
\end{abstract}

Conclusions: Our study suggests that three genetic variants, independent of their associations with age at menarche or age at natural menopause, were associated with breast cancer risk and may contribute modestly to breast cancer risk prediction; however, the combination of the 19 age at menarche or the 17 age at natural menopause associated SNPs did not appear to be useful for identifying a high risk subgroup for breast cancer.

\section{Introduction}

A younger age at menarche and an older age at menopause are well-established risk factors for the development of breast cancer [1]. In the general population, the risk of breast cancer decreases by $10 \%$ for each 2 -year

\footnotetext{
* Correspondence: chunhe@iupui.edu

'Department of Public Health, Indiana University School of Medicine, 980

West Walnut Street, R3-C241, Indianapolis, IN 46202, USA

Full list of author information is available at the end of the article
}

delay in menarche [2] but increases by $3 \%$ for each year that menopause is delayed [3]. These associations are consistent with the hypothesis that breast cancer risk is related to the extent of steroid hormone exposure during a woman's reproductive years, which drives breast mitotic activity and determines the probability of tumorigenic somatic events [4].

Recently, genome-wide association studies (GWAS) have identified several new common genetic loci

\section{Biomed Central}


associated with either age at menarche or age at natural menopause. Four independent GWAS of age at menarche have identified two novel loci at $L I N 28 B$ and $9 q 31.2$ [5-8], and two GWAS of age at natural menopause have identified four novel loci on chromosomes 5, 6, 19, and 20 [5,9]. Most recently, the ReproGen Consortium, which consisted of these initial GWASs and many additional studies, has conducted expanded metaanalyses for age at menarche [10] and age at natural menopause [11] and reported more new loci identified for each trait. Given the well-established associations of age at menarche and age at natural menopause with breast cancer risk, we set out to assess whether these common genetic loci influence breast cancer risk and whether a genetic risk score (GRS) for these reproductive events might be useful for identifying a high-risk subgroup for breast cancer. Furthermore, since the reproductive risk factors have been observed to be differentially associated with breast cancer by tumor histological subtypes [12-16], we assessed these genetic associations by tumor histological subtypes defined by estrogen receptor (ER) status.

We therefore conducted a meta-analysis of six population-based studies to investigate the association between genetic loci associated with age at menarche or age at natural menopause and breast cancer risk. We assessed 19 and 17 single-nucleotide polymorphisms (SNPs) that have been previously reported to be linked to age at menarche [10] and age at natural menopause [11], respectively, among up to 3,683 breast cancer cases and 34,174 controls in women of European ancestry and evaluated whether these SNPs were differentially associated with breast cancer subtypes defined by ER status in two studies in which such data were available.

\section{Materials and methods}

\section{Study population}

The ReproGen Consortium was formed by more than 30 studies in the US and Europe to investigate the genetics of reproductive aging traits [10,11]. Our analysis used data from six population-based studies from the ReproGen Consortium: the Nurses' Health Study (NHS), the Women's Genome Health Study (WGHS), the SardiNIA Breast Cancer Study (SardiNIA), the Rotterdam Study I and II (RSI+II), the Framingham Heart Study (FHS), and the Atherosclerosis Risk in Communities Study (ARIC). Each study had at least 200 breast cancer cases. Four studies were prospective cohort studies, one was a nested case-control study, and one was a case-control study. A description of the six studies is provided in Table 1, and more information is given in Additional file 1. Briefly, breast cancer cases occurring in defined populations during specific periods of time were identified by structured questionnaires, medical records, or linkage with a nationwide registry of cancer or death index or both. By the time we conducted this study, the majority of the women in these studies had passed through menopause. As most of the participants in these studies were European whites, we restricted analyses to women of European ancestry. We excluded subjects with missing information on age. Two studies (NHS and WGHS) provided information on the ER status of the breast tumors for a subset of the cases. This information was extracted from medical records. Each study was approved by the relevant local institutional review boards.

\section{Genotype data}

We analyzed genotypes for 19 and 17 independent SNPs with reported associations with age at menarche and age at natural menopause, respectively, in the ReproGen Consortium, in which all SNPs achieved genome-wide significance in the meta-analysis of each trait (combined stage 1 and replication $P$ value of less than $1 \times 10^{-8}$ ) $[10,11]$. None of these SNPs has been reported to be associated with breast cancer risk in previous GWAS and this is likely because of the very stringent $P$ value threshold used to declare genome-wide significance (usually, $P$ values were less than at least $1 \times 10^{-7}$ ). As positive controls, 10 SNPs with consistently reported

Table 1 List of participating studies and number of case and control subjects

\begin{tabular}{|c|c|c|c|c|c|c|}
\hline $\begin{array}{l}\text { Study } \\
\text { acronym }\end{array}$ & Study name & Study design & $\begin{array}{c}\text { Case } \\
\text { subjects } \\
(n=3,683)\end{array}$ & $\begin{array}{c}\mathrm{ER}^{+} / \mathrm{ER}^{-} \\
\text {subjects } \\
(n=1,716 / 371)\end{array}$ & $\begin{array}{c}\text { Control } \\
\text { subjects } \\
(n=34,174)\end{array}$ & $\begin{array}{c}\text { All subjects } \\
(n= \\
37,857)\end{array}$ \\
\hline NHS-BC & Nurses' Health Study-Breast Cancer & $\begin{array}{l}\text { Nested case- } \\
\text { control }\end{array}$ & $1,145(31.1)$ & $807 / 181$ & $1,142(3.3)$ & $2,287(6.0)$ \\
\hline WGHS & Women's Genome Health Study & Prospective cohort & $1,099(29.8)$ & $909 / 190$ & $22,205(65.0)$ & $23,304(61.5)$ \\
\hline SardBC & SardiNIA Breast Cancer Study & Case-control & $809(22.0)$ & - & $674(2.0)$ & $1,483(3.9)$ \\
\hline $\mathrm{RSI}+\|$ & Rotterdam Study I and II & Prospective cohort & $216(5.9)$ & - & $4,261(12.5)$ & $4,477(11.8)$ \\
\hline FHS & Framingham Heart Study & Prospective cohort & $207(5.6)$ & - & $3,698(10.8)$ & $3,905(10.3)$ \\
\hline ARIC & $\begin{array}{l}\text { Atherosclerosis Risk in Communities } \\
\text { Study }\end{array}$ & Prospective cohort & $207(5.6)$ & - & 2,194 (6.4) & $2,401(6.3)$ \\
\hline
\end{tabular}

Data are presented as the number (percentage) of cases, controls, and all subjects. ER, estrogen receptor. 
associations with breast cancer as shown in recent GWAS were included [17-19]. All 46 SNPs are listed in Table S1 of Additional file 2. Genotypes used in this analysis have been previously described $[10,11]$. Complete genotype data from a total of up to 3,683 cases and 34,174 control subjects were available for analysis after the exclusions described in the 'Study population' section.

\section{Breast cancer risk factors}

The six studies from the ReproGen Consortium provided information on one or more of the following risk factors for breast cancer: age (continuous, at study entry or diagnosis), age at menarche (continuous, between 9 and 17 years), age at natural menopause (continuous, between 40 and 60 years), age at first live birth (less than 20,20 to 24,25 to 29 or no birth, at least 30 years), family history of breast cancer in first-degree relatives (yes/no), alcohol consumption (less than 5, 5 to 15,15 to 30 , at least $30 \mathrm{~g} /$ day), parity ( 0,1 to 2 , at least $3)$, menopausal hormone therapy (ever/never), oral contraceptive (OC) use (ever/never), and adult body mass index (BMI) (continuous).

\section{Genetic risk score computation}

The GRS was calculated on the basis of the 19 and 17 independent SNPs identified in previous studies as being associated with age at menarche and age at natural menopause, respectively $[10,11]$. As a younger age at menarche and an older age at menopause are independently associated with an elevated breast cancer risk, we computed separate GRSs for a younger age at menarche and an older age at natural menopause. The risk allele was defined as an allele that was associated with a younger age at menarche or an older age at natural menopause. Two methods were used to determine the GRS: a simple count method (count GRS) and a weighted method (weighted GRS). We assumed an additive genetic model for each SNP, applying a linear weighting of 0,1 , or 2 to genotypes containing 0,1 , or 2 risk alleles, respectively. The count method assumes that each SNP contributes equally to the risk of breast cancer. The count GRS was calculated by simply summing the number of risk alleles of each SNP. For the weighted GRS, each SNP was weighted by $\beta$-coefficients obtained from the replication studies of recent meta-analyses of two traits $[10,11]$. The weighted GRS was calculated by multiplying each $\beta$-coefficient by the number of corresponding risk alleles $(0,1$, or 2$)$ and then summing the products. To simplify interpretation and facilitate comparison with the count GRS, the weighted GRS was further divided by twice the sum of the $\beta$-coefficients and then multiplied by the total number of risk alleles. To provide a positive control and also to control for potential confounding by known breast cancer-associated genetic variants, a count GRS was computed on the basis of the 10 SNPs with consistently reported associations with breast cancer [19]: rs2981582, rs3803662, rs11249433, rs7716600, rs13387042, rs889312, rs13281615, rs999737, rs3817198, and rs1045485.

\section{Statistical analysis}

In each of the six studies, we performed logistic regression to evaluate the association with breast cancer for each of the 46 candidate SNPs, assuming an additive genetic model. Logistic regression was also used to analyze the association between GRS and breast cancer by including both GRSs for age at menarche and age at natural menopause in the model as the main effects. The GRSs were modeled as continuous variables or categorized into quintiles, and the cutoff points for quintiles were based on the WGHS population, which is the largest prospective cohort population among all participating studies. This approach was applied to each of the six participating studies. Odds ratios (ORs) and 95\% confidence intervals (CIs) were estimated from logistic regression. To control for potential confounding by population stratification, we adjusted for the top principal components of genetic variation chosen for each study. We adjusted for age in the main model. To examine whether the genetic association of each of the candidate SNPs or GRSs with breast cancer is mediated through the onset of menarche or natural menopause, we then adjusted for self-reported age at menarche and age at natural menopause in the main model. Other conventional risk factors for breast cancer - including age at first live birth, family history of breast cancer in first-degree relatives, alcohol consumption, parity, menopausal hormone therapy, OC use, and adult BMI were further included in the model to control for potential confounding in studies which had such data available. To examine whether these genetic associations differ by breast cancer subtypes, in each of the two studies that provided information on ER status, we then investigated the genetic association of each of the candidate SNPs or GRSs with breast cancer in subgroup analysis by ER histological status (positive or negative).

Forest plots were used to present study-specific ORs and $95 \%$ CIs. We then performed meta-analyses by using the fixed-effects model to estimate summary ORs from study-specific estimatesthat were weighted by the inverse of the variance of each study. As the meta-analyses restricted to prospective cohort studies or casecontrol studies yielded similar results, we present results from only the meta-analysis of all six participating studies. We also tested the heterogeneity of associations across studies as well as across different tumor subtypes by using the Q test [20]. 
All statistical analyses were performed by using SAS version 9.1 software (SAS Institute Inc., Cary, NC, USA). Power calculations were carried out by using Quanto (University of Southern California, Los Angeles, CA, USA). All $P$ values were based on two-sided tests and were considered statistically significant if less than 0.05 . Because SNPs were selected on the basis of an $a$ priori hypothesis, adjustments for multiple comparison tests were not performed.

\section{Results}

The six participating studies contributed 3,683 breast cancer cases and 34,174 controls of self-reported white women of European ancestry (Table 1), all with available data on age and the 46 candidate SNPs, and at least one of the conventional risk factors considered. Of the 3,683 cases, about $52 \%$ were from the four prospective cohort studies (WGHS, RSI+II, FHS, and ARIC), about 30\% were from the nested case-control study in NHS, and about $18 \%$ were from the population-based case-control study in SardiNIA. ER status was known for 2,087 cases in the NHS and the WGHS. On average, compared with the controls, the cases had a younger age at menarche and an older age at natural menopause. The expected associations with breast cancer were generally observed for the conventional risk factors across all of the studies (Table S2 of Additional file 3). The associations of the 46 candidate SNPs with age at menarche or age at natural menopause in the six studies were consistent with the original findings from the two meta-analyses $[10,11]$.

Table 2 shows the risk allele frequency and the corresponding per-risk-allele OR of breast cancer for each of the 46 candidate SNPs. The results are arranged in order of the strength of statistical significance ( $P$ value). The allele frequency for each SNP in the controls was similar to those reported for populations of European descent [21-23]. After adjusting for age and potential population stratification, we found that, among the 19 candidate SNPs for a younger age at menarche, two SNPs, rs1079866 and rs7821178, were significantly associated with breast cancer risk and had corresponding per-risk-allele ORs of 1.14 (96\% CI $=1.05$ to $1.24 ; P$ value $=0.003 ; P$ for heterogeneity $=0.37)$ and $1.08(95 \%$ $\mathrm{CI}=1.02$ to $1.15 ; P$ value $=0.009 ; P$ for heterogeneity $=$ $0.43)$, respectively. The SNP rs1079866 is located about $250 \mathrm{~kb}$ away from the INHBA gene on chromosome 7, whereas SNP rs7821178 is about $181 \mathrm{~kb}$ away from the PXMP3 gene (also known as PEX3) on chromosome 8. The strongest GWAS hit for age at menarche, rs7759938 at LIN28B on chromosome 6, was not found to be associated with breast cancer risk $(P$ value $=0.60)$. Of the 17 candidate SNPs associated with an older age at natural menopause, one SNP, rs2517388, was significantly associated with breast cancer risk with a per-risk- allele OR of $1.10(95 \% \mathrm{CI}=1.01$ to $1.20 ; P$ value $=$ 0.023 ; $P$ for heterogeneity $=0.08$ ). This SNP is an intronic SNP in the $A S H 2 L$ gene on chromosome 8 . The study-specific and summary ORs for the three associated SNPs are shown in Figure 1. Further adjustment for conventional risk factors - including age at menarche, age at natural menopause, age at first live birth, family history of breast cancer in first-degree relatives, alcohol consumption, parity, menopausal hormone therapy, OC use, and adult BMI - did not change the results substantially. For candidate loci for age at menarche and age at natural menopause, the findings did not differ materially when we further adjusted for known breast cancer-associated SNPs.

To evaluate the combined effect of candidate SNPs on breast cancer risk, we calculated a GRS for each trait by using either a count GRS or a weighted GRS approach. The mean values of count and weighted GRSs were 20.41 and 20.03, respectively, for age at menarche and 16.21 and 14.32, respectively, for age at natural menopause (Table 3). Based on the count GRS for a younger age at menarche, the OR for breast cancer associated with each point scored, corresponding to 1 risk allele, was 1.01 (95\% CI $=1.00$ to 1.03$)$ after age and potential population stratification were adjusted for. ORs did not increase linearly across quintiles of GRS for age at menarche $(P$ for trend $=0.06)$. Compared with women in the lowest quintile, women in the fourth and fifth quintiles had ORs for breast cancer of 1.14 (95\% CI = 1.01 to 1.28$)$ and $1.13(95 \% \mathrm{CI}=1.00$ to 1.27$)$, respectively. Results were similar when analyses were performed by using weighted GRS. Overall, we did not observe statistically significant associations between breast cancer risk and age at natural menopause when either count or weighted GRS was used.

In secondary analyses, we then determined whether the associations of the 46 candidate SNPs with breast cancer vary across tumor subtypes defined by ER status in the NHS and the WGHS (Table 4). For the two SNPs (rs1079866 and rs7821178) that had reported associations with age at menarche and that were associated with overall breast cancer risk, we found no statistically significant evidence that the associations differed across subtypes ( $P$ for heterogeneity $=0.31$ and 0.66 , respectively), although rs1079866 appeared to have a stronger association with $\mathrm{ER}^{+}$tumors (per-allele $\mathrm{OR}=1.26$; $95 \%$ $\mathrm{CI}=1.12$ to 1.41 ) than with $\mathrm{ER}^{-}$tumors (per-allele OR $=1.11 ; 95 \% \mathrm{CI}=0.89$ to 1.38 ). Of note, one SNP that had a reported association with age at menarche, rs17188434, had a significantly stronger association with $\mathrm{ER}^{-}$tumors (per-allele $\mathrm{OR}=1.51 ; 95 \% \mathrm{CI}=1.15$ to 1.98) than with $\mathrm{ER}^{+}$tumors (per-allele $\mathrm{OR}=1.08$; $95 \%$ $\mathrm{CI}=0.92$ to 1.26 ; $P$ for heterogeneity $=0.035$ ). Another SNP that had a reported association with age at 


\begin{tabular}{|c|c|c|c|c|c|c|c|c|c|c|c|c|c|}
\hline SNP & Gene(s) $)^{a}$ & $\begin{array}{l}\text { Distance } \\
\text { from gene }\end{array}$ & Chromosome & $\begin{array}{l}\text { Position } \\
\text { (Build 36) }\end{array}$ & $\begin{array}{l}\text { Risk/Reference } \\
\text { alleles }^{\text {b }}\end{array}$ & $\begin{array}{l}\text { Effect } \\
\text { allele }\end{array}$ & $\begin{array}{l}\text { Allele frequencyc of } \\
\text { cases/controls }^{\mathrm{d}}\end{array}$ & Effect & SE & $\begin{array}{l}\text { OR }(95 \% \\
C I)^{\mathrm{e}}\end{array}$ & $\begin{array}{c}P \\
\text { value }^{f}\end{array}$ & Direction $^{g}$ & $\begin{array}{c}P \text { value for } \\
\text { heterogeneity }\end{array}$ \\
\hline \multicolumn{14}{|c|}{ Age at menarche } \\
\hline rs1079866 & INHBA & $\sim 250 \mathrm{~kb}$ & 7 & 41436618 & $C / G$ & c & $0.87 / 0.85$ & 0.132 & 0.044 & $\begin{array}{c}1.14 \\
(1.05-1.24)\end{array}$ & 0.003 & ++-+- & 0.37 \\
\hline rs7821178 & PXMP3 & $\sim 181$ kb & 8 & 78256392 & $\mathrm{~A} / \mathrm{C}$ & a & $0.35 / 0.33$ & 0.081 & 0.031 & $\begin{array}{c}1.08 \\
(1.02-1.15)\end{array}$ & 0.009 & ++-++ & 0.43 \\
\hline rs7642134 & VGLL3 & $\sim 70 \mathrm{~kb}$ & 3 & 86999572 & $A / G$ & a & $0.39 / 0.38$ & 0.058 & 0.03 & $\begin{array}{c}1.06 \\
(1.00-1.12)\end{array}$ & 0.056 & ++++-+ & 0.83 \\
\hline rs10980926 & ZNF483 & Intronic & 9 & 113333455 & $\mathrm{G} / \mathrm{A}$ & g & $0.64 / 0.65$ & -0.057 & 0.031 & $\begin{array}{c}0.94 \\
(0.89-1.00)\end{array}$ & 0.066 & -+- & 0.58 \\
\hline rs1398217 & FUSSEL18 & Intronic & 18 & 43006236 & $\mathrm{G} / \mathrm{C}$ & g & $0.42 / 0.42$ & 0.048 & 0.03 & $\begin{array}{c}1.05 \\
(0.99-1.11)\end{array}$ & 0.10 & ++-+++ & 0.40 \\
\hline rs17188434 & $N R 4 A 2$ & $\sim 84 \mathrm{~kb}$ & 2 & 156805022 & $C / T$ & c & $0.07 / 0.07$ & 0.075 & 0.059 & $\begin{array}{c}1.08 \\
(0.96-1.21)\end{array}$ & 0.20 & +++- & 0.34 \\
\hline rs13187289 & PHF15 & $\sim 12 \mathrm{~kb}$ & 5 & 133877076 & $C / G$ & c & $0.80 / 0.80$ & -0.038 & 0.038 & $\begin{array}{c}0.96 \\
(0.89-1.04)\end{array}$ & 0.32 & -+-+ & 0.03 \\
\hline rs12617311 & PLCL1 & $\sim 195$ kb & 2 & 199340810 & $\mathrm{~A} / \mathrm{G}$ & a & $0.32 / 0.32$ & -0.032 & 0.033 & $\begin{array}{c}0.97 \\
(0.91-1.03)\end{array}$ & 0.33 & -+-+- & 0.81 \\
\hline rs17268785 & CCDC85A & Intronic & 2 & 56445587 & $\mathrm{~A} / \mathrm{G}$ & a & $0.84 / 0.84$ & 0.037 & 0.041 & $\begin{array}{c}1.04 \\
(0.96-1.12)\end{array}$ & 0.37 & -+-+++ & 0.89 \\
\hline rs2002675 & $\begin{array}{l}\text { TRA2B, } \\
\text { ETV5 }\end{array}$ & $\begin{array}{c}\sim 4 \mathrm{~kb}, \sim 135 \\
\mathrm{~kb}\end{array}$ & 3 & 187112262 & $\mathrm{~A} / \mathrm{G}$ & a & $0.58 / 0.57$ & 0.022 & 0.03 & $\begin{array}{c}1.02 \\
(0.96-1.08)\end{array}$ & 0.47 & ++++- & 0.92 \\
\hline rs466639 & $R X R G$ & Intronic & 1 & 163661506 & $\mathrm{~T} / \mathrm{C}$ & t & $0.12 / 0.12$ & -0.032 & 0.046 & $\begin{array}{c}0.97 \\
(0.89-1.06)\end{array}$ & 0.49 & -+-+- & 0.35 \\
\hline rs1659127 & $M K L 2$ & $\sim 28 \mathrm{~kb}$ & 16 & 14295806 & $\mathrm{G} / \mathrm{A}$ & g & $0.67 / 0.66$ & 0.022 & 0.033 & $\begin{array}{c}1.02 \\
(0.96-1.09)\end{array}$ & 0.50 & +++++- & 0.28 \\
\hline rs9635759 & CA10 & $\sim 94 \mathrm{~kb}$ & 17 & 46968784 & $\mathrm{G} / \mathrm{A}$ & g & 0.69/0.70 & -0.021 & 0.034 & $\begin{array}{c}0.98 \\
(0.92-1.05)\end{array}$ & 0.54 & -+-+- & 0.32 \\
\hline rs10899489 & GAB2 & Intronic & 11 & 77773021 & $C / A$ & c & $0.85 / 0.84$ & 0.025 & 0.041 & $\begin{array}{c}1.03 \\
(0.95-1.11)\end{array}$ & 0.54 & ++++- & 0.97 \\
\hline rs10423674 & CRTC1 & Intronic & 19 & 18678903 & $C / A$ & c & $0.67 / 0.67$ & 0.017 & 0.032 & $\begin{array}{c}1.02 \\
(0.96-1.08)\end{array}$ & 0.59 & +++-+- & 0.60 \\
\hline rs7759938 & LIN28B & $\sim 26 \mathrm{~kb}$ & 6 & 105485647 & $\mathrm{~T} / \mathrm{C}$ & t & $0.69 / 0.69$ & -0.017 & 0.032 & $\begin{array}{c}0.98 \\
(0.92-1.05)\end{array}$ & 0.60 & +-+- & 0.53 \\
\hline rs2090409 & TMEMЗ8B & $\sim 400 \mathrm{~kb}$ & 9 & 108006909 & $\mathrm{~A} / \mathrm{C}$ & a & $0.33 / 0.33$ & 0.012 & 0.031 & $\begin{array}{c}1.01 \\
(0.95-1.08)\end{array}$ & 0.69 & -++-+ & 0.21 \\
\hline rs6438424 & 3913.32 & Intergenic & 3 & 119057512 & $\mathrm{~A} / \mathrm{C}$ & a & $0.50 / 0.50$ & 0.002 & 0.029 & $\begin{array}{c}1.00 \\
(0.95-1.06)\end{array}$ & 0.94 & -+++ & 0.88 \\
\hline rs6589964 & $B S X$ & $\sim 18 \mathrm{~kb}$ & 11 & 122375893 & $\mathrm{~A} / \mathrm{C}$ & a & $0.48 / 0.48$ & -0.001 & 0.031 & $\begin{array}{c}1.00 \\
(0.94-1.06)\end{array}$ & 0.99 & -++++ & 0.20 \\
\hline \multicolumn{14}{|c|}{ Age at natural menopause } \\
\hline rs2517388 & $A S H 2 L$ & Intronic & 8 & 38096889 & $\mathrm{G} / \mathrm{T}$ & g & $0.17 / 0.16$ & 0.096 & 0.042 & $\begin{array}{c}1.10 \\
(1.01-1.20)\end{array}$ & 0.023 & +++-+- & 0.08 \\
\hline
\end{tabular}


Table 2 Association of candidate single-nucleotide polymorphism loci and the risk of breast cancer (Continued)

\begin{tabular}{|c|c|c|c|c|c|c|c|c|c|c|c|c|c|}
\hline rs4693089 & HEL308 & Intronic & 4 & 84592646 & $\mathrm{G} / \mathrm{A}$ & g & $0.47 / 0.48$ & -0.06 & 0.031 & $\begin{array}{c}0.94 \\
(0.89-1.00)\end{array}$ & 0.054 & -—— & 0.38 \\
\hline rs11668344 & TMEM150B & Intronic & 19 & 60525476 & $A / G$ & a & $0.64 / 0.64$ & -0.049 & 0.03 & $\begin{array}{c}0.95 \\
(0.90-1.01)\end{array}$ & 0.11 & +--- & 0.02 \\
\hline rs2307449 & POLG & Intronic & 15 & 87664932 & $T / G$ & $\mathrm{t}$ & $0.60 / 0.61$ & -0.045 & 0.031 & $\begin{array}{c}0.96 \\
(0.90-1.02)\end{array}$ & 0.15 & -+-+- & 0.20 \\
\hline rs16991615 & MCM8 & Missense & 20 & 5896227 & $A / G$ & a & $0.07 / 0.06$ & 0.079 & 0.06 & $\begin{array}{c}1.08 \\
(0.96-1.22)\end{array}$ & 0.18 & +++-+ & 0.80 \\
\hline rs12294104 & $\begin{array}{c}\text { C11orf46, } \\
\text { PPED2 }\end{array}$ & $\sim 24 \mathrm{~kb}, \sim 49 \mathrm{~kb}$ & 11 & 30339475 & $\mathrm{~T} / \mathrm{C}$ & $\mathrm{t}$ & $0.17 / 0.17$ & 0.046 & 0.039 & $\begin{array}{c}1.05 \\
(0.97-1.13)\end{array}$ & 0.24 & ++-++- & 0.56 \\
\hline rs12461110 & NLRP11 & Missense & 19 & 61012475 & $\mathrm{G} / \mathrm{A}$ & g & $0.64 / 0.65$ & -0.036 & 0.031 & $\begin{array}{c}0.96 \\
(0.91-1.03)\end{array}$ & 0.24 & -++-+ & 0.32 \\
\hline rs2303369 & FNDC4 & Intronic & 2 & 27568920 & $C / T$ & c & $0.62 / 0.63$ & -0.031 & 0.03 & $\begin{array}{c}0.97 \\
(0.91-1.03)\end{array}$ & 0.31 & -++-+ & 0.22 \\
\hline rs4886238 & TDRD3 & Intronic & 13 & 60011740 & $A / G$ & a & $0.35 / 0.34$ & 0.029 & 0.031 & $\begin{array}{c}1.03 \\
(0.97-1.09)\end{array}$ & 0.35 & ++-- & 0.79 \\
\hline rs2277339 & PRIM1 & Missense & 12 & 55432336 & $\mathrm{~T} / \mathrm{G}$ & $t$ & 0.90/0.89 & 0.037 & 0.05 & $\begin{array}{c}1.04 \\
(0.94-1.14)\end{array}$ & 0.46 & ++++- & 0.47 \\
\hline rs10852344 & $\begin{array}{c}\text { GSPT1, } \\
\text { TNFRSF17 }\end{array}$ & $\sim 7 \mathrm{~kb}, \sim 42 \mathrm{~kb}$ & 16 & 11924420 & $C / T$ & c & $0.41 / 0.41$ & 0.020 & 0.030 & $\begin{array}{c}1.02 \\
(0.96-1.08)\end{array}$ & 0.50 & ++-++ & 0.87 \\
\hline rs10183486 & TLK1 & Intronic & 2 & 171699217 & $C / T$ & c & $0.62 / 0.62$ & -0.015 & 0.031 & $\begin{array}{c}0.99 \\
(0.93-1.05)\end{array}$ & 0.63 & -+-+ & 0.98 \\
\hline rs4246511 & RHBDL2 & Intronic & 1 & 39152972 & $\mathrm{~T} / \mathrm{C}$ & $\mathrm{t}$ & $0.27 / 0.27$ & 0.016 & 0.035 & $\begin{array}{c}1.02 \\
(0.95-1.09)\end{array}$ & 0.66 & ++-+ & 0.69 \\
\hline rs2153157 & SYCP2L & Intronic & 6 & 11005474 & $A / G$ & a & $0.50 / 0.50$ & 0.011 & 0.029 & $\begin{array}{c}1.01 \\
(0.96-1.07)\end{array}$ & 0.70 & ++-+-+ & 0.59 \\
\hline rs1635501 & EXO1 & Intronic & 1 & 240107398 & $\mathrm{~T} / \mathrm{C}$ & $\mathrm{t}$ & $0.53 / 0.52$ & 0.011 & 0.034 & $\begin{array}{c}1.01 \\
(0.95-1.08)\end{array}$ & 0.74 & +-+-+ & 0.31 \\
\hline rs365132 & UIMC1 & Synonymous & 5 & 176311180 & $\mathrm{~T} / \mathrm{G}$ & $\mathrm{t}$ & $0.50 / 0.50$ & -0.009 & 0.03 & $\begin{array}{c}0.99 \\
(0.93-1.05)\end{array}$ & 0.76 & +-+-+ & 0.58 \\
\hline rs1046089 & BAT2 & Missense & 6 & 31710946 & $\mathrm{G} / \mathrm{A}$ & g & $0.65 / 0.65$ & -0.008 & 0.031 & $\begin{array}{c}0.99 \\
(0.93-1.05)\end{array}$ & 0.79 & -+-+-+ & 0.24 \\
\hline \multicolumn{14}{|l|}{ Breast cancer } \\
\hline rs2981582 & FGFR2 & Intronic & 10 & 123342307 & $A / G$ & a & $0.44 / 0.38$ & 0.188 & 0.03 & $\begin{array}{c}1.21 \\
(1.14-1.28)\end{array}$ & $\begin{array}{l}4.7 \times \\
10^{-10}\end{array}$ & ++++++ & 0.10 \\
\hline rs3803662 & TOX3 & $\sim 6 \mathrm{~kb}$ & 16 & 51143842 & $A / G$ & $a$ & $0.31 / 0.25$ & 0.166 & 0.032 & $\begin{array}{c}1.18 \\
(1.11-1.26)\end{array}$ & $\begin{array}{l}2.6 \times \\
10^{-7}\end{array}$ & ++++-+ & 0.31 \\
\hline rs11249433 & FCGRIB & $\sim 245 \mathrm{~kb}$ & 1 & 120982136 & $\mathrm{G} / \mathrm{A}$ & g & $0.44 / 0.46$ & 0.149 & 0.031 & $\begin{array}{c}1.16 \\
(1.09-1.23)\end{array}$ & $\begin{array}{l}1.9 \times \\
10^{-6}\end{array}$ & +++++- & 0.26 \\
\hline rs7716600 & MRPS30 & $\sim 59 \mathrm{~kb}$ & 5 & 44910762 & $\mathrm{~A} / \mathrm{C}$ & a & $0.24 / 0.20$ & 0.148 & 0.035 & $\begin{array}{c}1.16 \\
(1.08-1.24)\end{array}$ & $\begin{array}{l}2.3 \times \\
10^{-5}\end{array}$ & ++++++ & 0.80 \\
\hline rs13387042 & TNP1 & $\sim 181 \mathrm{~kb}$ & 2 & 217614077 & $A / G$ & $a$ & $0.55 / 0.50$ & 0.114 & 0.029 & $\begin{array}{c}1.12 \\
(1.06-1.19)\end{array}$ & $\begin{array}{l}1.0 \times \\
10^{-4}\end{array}$ & ++-+++ & 0.20 \\
\hline rs889312 & MAP3K1 & $\sim 43 \mathrm{~kb}$ & 5 & 56067641 & $C / A$ & c & $0.30 / 0.32$ & 0.122 & 0.032 & $\begin{array}{c}1.13 \\
(1.06-1.20)\end{array}$ & $\begin{array}{l}1.5 \times \\
10^{-4}\end{array}$ & ++++++ & 0.65 \\
\hline
\end{tabular}


Table 2 Association of candidate single-nucleotide polymorphism loci and the risk of breast cancer (Continued)

\begin{tabular}{|c|c|c|c|c|c|c|c|c|c|c|c|c|c|}
\hline rs13281615 & 8924.21 & Intergenic & 8 & 128424800 & $\mathrm{G} / \mathrm{A}$ & g & $0.43 / 0.50$ & 0.107 & 0.03 & $\begin{array}{c}1.11 \\
(1.05-1.18)\end{array}$ & $\begin{array}{l}3.0 \times \\
10^{-4}\end{array}$ & ++++- & 0.01 \\
\hline rs999737 & RAD51L1 & Intronic & 14 & 68104435 & $C / T$ & c & $0.77 / 0.80$ & 0.079 & 0.035 & $\begin{array}{c}1.08 \\
(1.01-1.16)\end{array}$ & 0.024 & ++++-+ & 0.31 \\
\hline rs3817198 & LSP1 & Intronic & 11 & 1865582 & $C / T$ & c & $0.32 / 0.34$ & 0.049 & 0.032 & $\begin{array}{c}1.05 \\
(0.99-1.12)\end{array}$ & 0.13 & +++-++ & 0.45 \\
\hline rs1045485 & CASP8 & Missense & 2 & 201857834 & $\mathrm{G} / \mathrm{C}$ & g & $0.88 / 0.92$ & 0.067 & 0.045 & $\begin{array}{c}1.07 \\
(0.98-1.17)\end{array}$ & 0.13 & ++-++ & 0.75 \\
\hline
\end{tabular}

${ }^{a}$ Nearest gene(s); ${ }^{b}$ the risk allele refers to the allele associated with a younger age at menarche, an older age at natural menopause, or an increased risk of breast cancer; ${ }^{c}$ effect allele frequency; ${ }^{\text {deffect allele }}$

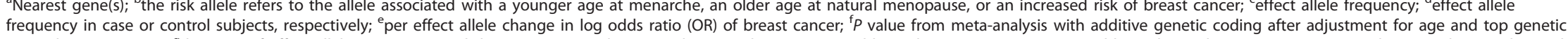
principle components; ${ }^{9}$ direction of effect allele association with breast cancer in the six studies in order: Nurses' Health Study, Women's Genome Health Study, SardiNIA Breast Cancer Study, Rotterdam Study I and

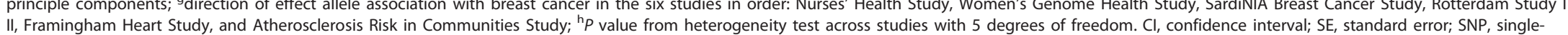
nucleotide polymorphism. 


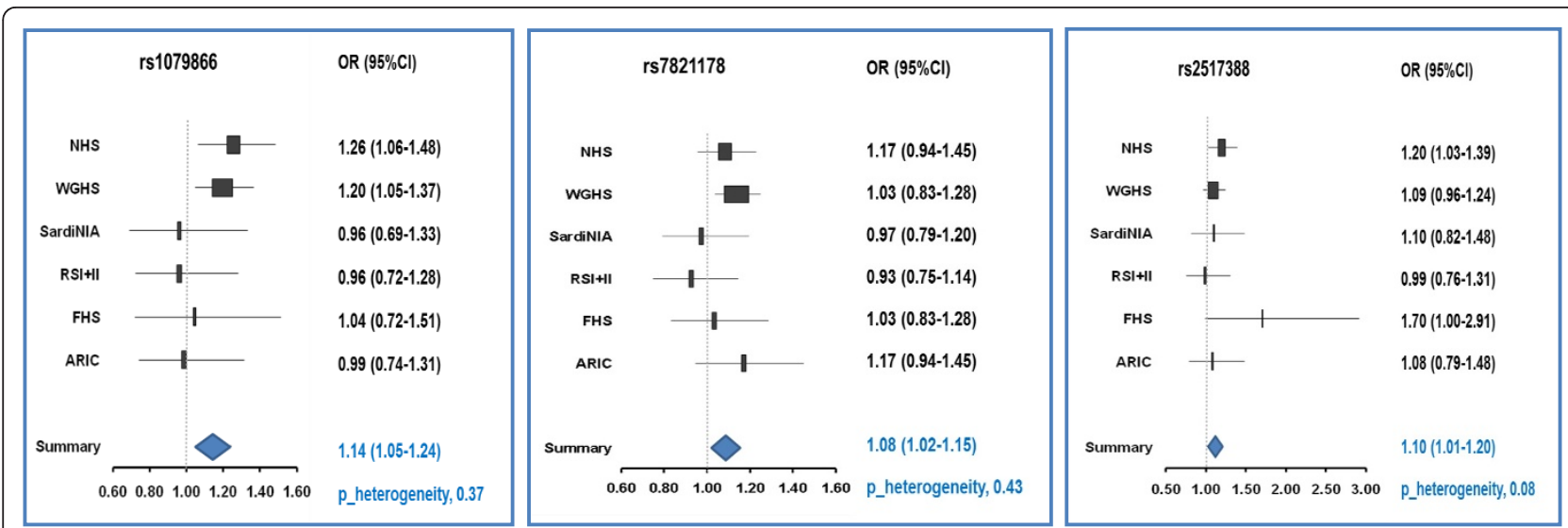

Figure 1 Forest plots for the three candidate loci (rs1079866, rs7821178, and rs2517388) in association with breast cancer risk. Perrisk-allele odds ratios (ORs) and 95\% confidence intervals (Cls) were obtained from unconditional logistic regression in each study, and age and potential population stratification were adjusted for. The size of the box is inversely proportional to the standard error of the log OR estimate. $P$ values for heterogeneity across studies are $0.37,0.43$, and 0.08, respectively. ARIC, Atherosclerosis Risk in Communities Study; FHS, Framingham Heart Study; NHS, Nurses' Health Study; RSI+II, Rotterdam Study I, II; SardiNIA, SardiNIA Breast Cancer Study; WGHS, Women's Genome Health Study.

Table 3 Association between genetic risk score and risk of breast cancer

\begin{tabular}{|c|c|c|c|c|c|c|c|}
\hline & \multicolumn{7}{|c|}{ Quintile of GRS } \\
\hline & $\begin{array}{l}\text { Continuous } \\
\text { GRS }\end{array}$ & Quintile 1 & Quintile 2 & Quintile 3 & Quintile 4 & Quintile 5 & $\begin{array}{l}P \text { for } \\
\text { trend }\end{array}$ \\
\hline \multicolumn{8}{|l|}{$\overline{\text { Count GRS }}{ }^{\mathrm{a}}$} \\
\hline \multicolumn{8}{|l|}{ Age at menarche } \\
\hline Cases/Controls & $3,683 / 34,174$ & $677 / 6,694$ & $739 / 6,814$ & $731 / 6,788$ & $783 / 6,915$ & $753 / 6,963$ & \\
\hline 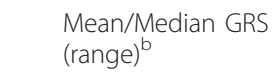 & $\begin{array}{l}20.41(9.12- \\
31.95)\end{array}$ & $\begin{array}{c}17.00(9.12- \\
18.09)\end{array}$ & $\begin{array}{c}19.02(18.09- \\
19.86)\end{array}$ & $\begin{array}{c}20.37(19.86- \\
21.05)\end{array}$ & $\begin{array}{c}21.90(21.05- \\
22.74)\end{array}$ & $\begin{array}{c}23.89(22.74- \\
31.95)\end{array}$ & \\
\hline OR $(95 \% \mathrm{Cl})^{c}$ & $1.01(1.00-1.03)$ & 1.00 & $1.10(0.97-1.24)$ & $1.01(0.89-1.14)$ & $1.14(1.01-1.28)$ & $1.13(1.00-1.27)$ & 0.06 \\
\hline \multicolumn{8}{|l|}{ Age at natural menopause } \\
\hline Cases/Controls & $3,683 / 34,174$ & $740 / 6,699$ & $751 / 6,948$ & $719 / 6,787$ & $755 / 6,929$ & $718 / 6,811$ & \\
\hline $\begin{array}{l}\text { Mean/Median GRS } \\
(\text { range })^{b}\end{array}$ & $\begin{array}{l}16.21(4.15- \\
26.52)\end{array}$ & $\begin{array}{l}12.95(4.15- \\
14.01)\end{array}$ & $\begin{array}{c}14.91(14.01- \\
15.62)\end{array}$ & $\begin{array}{c}16.15(15.62- \\
16.90)\end{array}$ & $\begin{array}{c}17.64(16.90- \\
18.37)\end{array}$ & $\begin{array}{c}19.55(18.37- \\
26.52)\end{array}$ & \\
\hline OR $(95 \% \mathrm{Cl})^{\mathrm{C}}$ & $1.00(0.98-1.01)$ & 1.00 & $1.02(0.90-1.15)$ & $0.93(0.82-1.05)$ & $1.02(0.90-1.14)$ & $0.98(0.87-1.11)$ & 0.54 \\
\hline \multicolumn{8}{|l|}{ Breast cancer } \\
\hline Cases/Controls & $3,683 / 34,174$ & $537 / 6,864$ & $542 / 6,525$ & $660 / 6,716$ & $956 / 7,352$ & $988 / 6,717$ & \\
\hline 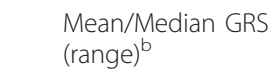 & $\begin{array}{c}8.88(1.06- \\
16.96)\end{array}$ & $6.04(1.06-7.01)$ & $7.99(7.01-8.05)$ & $8.99(8.05-9.07)$ & $9.99(9.07-10.86)$ & $\begin{array}{c}11.31(10.86- \\
16.96)\end{array}$ & \\
\hline OR $(95 \% \mathrm{Cl})^{\mathrm{c}}$ & $1.13(1.11-1.15)$ & 1.00 & $1.19(1.04-1.37)$ & $1.38(1.21-1.57)$ & $1.60(1.41-1.81)$ & $1.89(1.67-2.14)$ & $1.5 \times 10^{-31}$ \\
\hline \multicolumn{8}{|l|}{ Weighted GRS ${ }^{a}$} \\
\hline \multicolumn{8}{|l|}{ Age at menarche } \\
\hline Cases/Controls & $3,683 / 34,174$ & $762 / 8,961$ & $711 / 6,811$ & $752 / 6,287$ & $728 / 6,059$ & $736 / 6,056$ & \\
\hline 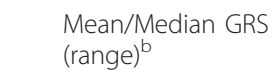 & $\begin{array}{c}20.03(7.15- \\
32.17)\end{array}$ & $\begin{array}{l}16.12(7.15- \\
17.50)\end{array}$ & $\begin{array}{c}18.47(17.50- \\
19.31)\end{array}$ & $\begin{array}{c}20.07(19.31- \\
20.83)\end{array}$ & $\begin{array}{c}21.63(20.83- \\
22.55)\end{array}$ & $\begin{array}{c}23.82(22.55- \\
32.17)\end{array}$ & \\
\hline OR $(95 \% \mathrm{Cl})^{\mathrm{C}}$ & $1.01(1.00-1.03)$ & 1.00 & $1.02(0.90-1.15)$ & $0.99(0.88-1.13)$ & $1.02(0.90-1.16)$ & $1.11(0.98-1.26)$ & 0.12 \\
\hline \multicolumn{8}{|c|}{ Age at natural menopause } \\
\hline Cases/Controls & $3,683 / 34,174$ & $749 / 7,038$ & $728 / 6,915$ & $756 / 6,749$ & $696 / 6,668$ & $755 / 6,804$ & \\
\hline 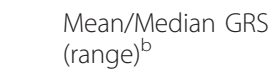 & $\begin{array}{l}14.32(2.78- \\
26.21)\end{array}$ & $\begin{array}{l}11.13(2.78- \\
12.18)\end{array}$ & $\begin{array}{c}12.96(12.18- \\
13.61)\end{array}$ & $\begin{array}{c}14.24(13.61- \\
14.88)\end{array}$ & $\begin{array}{c}15.57(14.88- \\
16.39)\end{array}$ & $\begin{array}{c}17.63(16.39- \\
26.21)\end{array}$ & \\
\hline OR $(95 \% \mathrm{CI})^{c}$ & $1.00(0.98-1.01)$ & 1.00 & $1.07(0.95-1.20)$ & $0.97(0.86-1.09)$ & $1.00(0.88-1.13)$ & $1.03(0.91-1.16)$ & 0.73 \\
\hline
\end{tabular}

${ }^{a}$ See the 'Materials and methods' section for count genetic risk score (GRS) and weighted GRS computation; ${ }^{b}$ mean for continuous GRS and median for each quintile; ${ }^{C}$ adjusted for age and potential population stratification. $\mathrm{Cl}$, confidence interval; OR, odds ratio. 
Table 4 Association of candidate single-nucleotide polymorphism loci and risk of breast cancer by estrogen receptor status in the Nurses' Health Study and Women's Genome Health Study

\begin{tabular}{|c|c|c|c|c|c|c|c|}
\hline \multirow[t]{2}{*}{ SNP } & \multicolumn{2}{|c|}{ All cases } & \multicolumn{2}{|l|}{$\mathrm{ER}^{+}$} & \multicolumn{2}{|l|}{$\mathrm{ER}^{-}$} & \multirow{2}{*}{$\begin{array}{c}\mathrm{ER}^{+} / \mathrm{ER}^{-} \\
P \text { value for heterogeneity }\end{array}$} \\
\hline & OR $(95 \% \mathrm{Cl})^{\mathrm{a}}$ & $P$ value $^{b}$ & OR $(95 \% \mathrm{Cl})^{\mathrm{a}}$ & $P$ value ${ }^{\mathrm{b}}$ & OR $(95 \% \mathrm{Cl})^{\mathrm{a}}$ & $P$ value ${ }^{\mathrm{b}}$ & \\
\hline Cases/Controls $^{d}$ & \multicolumn{2}{|c|}{$2,087 / 23,319$} & \multicolumn{2}{|c|}{$1,716 / 23,319$} & \multicolumn{2}{|c|}{$371 / 23,319$} & \\
\hline \multicolumn{8}{|l|}{ Age at menarche } \\
\hline rs1079866 & $1.22(1.10-1.35)$ & $2.0 \times 10^{-4}$ & $1.26(1.12-1.41)$ & $7.3 \times 10^{-5}$ & $1.11(0.89-1.38)$ & 0.34 & 0.31 \\
\hline rs7821178 & $1.12(1.04-1.20)$ & $3.3 \times 10^{-3}$ & $1.10(1.01-1.19)$ & 0.025 & $1.14(0.98-1.34)$ & 0.10 & 0.66 \\
\hline rs17188434 & $1.15(1.00-1.33)$ & 0.048 & $1.08(0.92-1.26)$ & 0.34 & $1.51(1.15-1.98)$ & $2.7 \times 10^{-3}$ & 0.035 \\
\hline rs13187289 & $0.92(0.84-1.00)$ & 0.055 & $0.90(0.82-0.99)$ & 0.026 & $1.05(0.87-1.28)$ & 0.60 & 0.15 \\
\hline rs10980926 & $0.93(0.87-1.00)$ & 0.067 & $0.94(0.87-1.02)$ & 0.14 & $0.90(0.77-1.05)$ & 0.17 & 0.58 \\
\hline rs7642134 & $1.06(0.99-1.14)$ & 0.12 & $1.07(0.99-1.16)$ & 0.089 & $1.09(0.93-1.27)$ & 0.28 & 0.86 \\
\hline rs10423674 & $1.05(0.97-1.13)$ & 0.22 & $1.06(0.97-1.15)$ & 0.19 & $0.95(0.81-1.11)$ & 0.50 & 0.23 \\
\hline rs6589964 & $0.95(0.89-1.03)$ & 0.22 & $0.94(0.87-1.02)$ & 0.13 & $1.00(0.85-1.17)$ & 0.98 & 0.51 \\
\hline rs1398217 & $1.04(0.97-1.12)$ & 0.25 & $1.02(0.95-1.10)$ & 0.57 & $1.11(0.95-1.29)$ & 0.19 & 0.36 \\
\hline rs1659127 & $1.03(0.96-1.12)$ & 0.41 & $1.00(0.92-1.09)$ & 0.98 & $1.09(0.92-1.29)$ & 0.32 & 0.38 \\
\hline rs12617311 & $0.97(0.89-1.05)$ & 0.44 & $1.00(0.91-1.09)$ & 0.93 & $0.94(0.79-1.12)$ & 0.48 & 0.56 \\
\hline rs17268785 & $1.04(0.94-1.14)$ & 0.48 & $1.07(0.96-1.19)$ & 0.22 & $0.83(0.68-1.00)$ & 0.055 & 0.023 \\
\hline rs2090409 & $0.97(0.90-1.05)$ & 0.50 & $0.96(0.89-1.05)$ & 0.38 & $0.98(0.84-1.15)$ & 0.82 & 0.85 \\
\hline rs10899489 & $1.03(0.93-1.14)$ & 0.52 & $1.02(0.92-1.14)$ & 0.67 & $0.98(0.79-1.21)$ & 0.86 & 0.72 \\
\hline rs6438424 & $0.98(0.91-1.05)$ & 0.55 & $0.99(0.92-1.07)$ & 0.79 & $0.98(0.84-1.14)$ & 0.76 & 0.88 \\
\hline rs2002675 & $1.02(0.95-1.10)$ & 0.55 & $1.04(0.96-1.12)$ & 0.35 & $0.94(0.81-1.10)$ & 0.45 & 0.27 \\
\hline rs466639 & $1.02(0.92-1.14)$ & 0.73 & $1.05(0.94-1.18)$ & 0.39 & $0.94(0.74-1.20)$ & 0.62 & 0.41 \\
\hline rs7759938 & 1.01 (0.93-1.09) & 0.84 & $1.02(0.94-1.11)$ & 0.63 & $1.00(0.84-1.17)$ & 0.96 & 0.80 \\
\hline rs9635759 & 1.01 (0.93-1.09) & 0.88 & $1.00(0.92-1.09)$ & 0.98 & $1.03(0.87-1.22)$ & 0.72 & 0.74 \\
\hline \multicolumn{8}{|c|}{ Age at natural menopause } \\
\hline rs2517388 & $1.14(1.03-1.25)$ & 0.010 & $1.11(1.00-1.24)$ & 0.046 & $1.32(1.09-1.61)$ & $4.7 \times 10^{-3}$ & 0.12 \\
\hline rs2303369 & $0.93(0.86-1.00)$ & 0.036 & $0.93(0.86-1.01)$ & 0.076 & $0.94(0.80-1.09)$ & 0.42 & 0.94 \\
\hline rs12461110 & $0.93(0.86-1.00)$ & 0.042 & $0.91(0.84-0.99)$ & 0.022 & $0.95(0.81-1.11)$ & 0.50 & 0.66 \\
\hline rs4886238 & $1.06(0.99-1.14)$ & 0.11 & $1.06(0.98-1.15)$ & 0.14 & $1.03(0.88-1.21)$ & 0.68 & 0.76 \\
\hline rs16991615 & $1.09(0.94-1.26)$ & 0.23 & $1.13(0.97-1.32)$ & 0.12 & $0.99(0.71-1.37)$ & 0.94 & 0.46 \\
\hline rs2277339 & $1.07(0.95-1.20)$ & 0.28 & $1.09(0.96-1.24)$ & 0.21 & $1.02(0.80-1.31)$ & 0.85 & 0.68 \\
\hline rs12294104 & $1.04(0.95-1.14)$ & 0.41 & $1.06(0.96-1.17)$ & 0.28 & $0.98(0.80-1.21)$ & 0.89 & 0.54 \\
\hline rs4246511 & $1.03(0.95-1.12)$ & 0.42 & $1.05(0.96-1.15)$ & 0.29 & $0.95(0.79-1.14)$ & 0.59 & 0.34 \\
\hline rs10852344 & $1.03(0.96-1.10)$ & 0.42 & $1.04(0.96-1.12)$ & 0.31 & $0.99(0.85-1.15)$ & 0.88 & 0.56 \\
\hline rs2153157 & $1.03(0.96-1.10)$ & 0.45 & $1.04(0.96-1.12)$ & 0.32 & $1.00(0.87-1.17)$ & 0.95 & 0.69 \\
\hline rs4693089 & $0.98(0.91-1.05)$ & 0.54 & $0.98(0.91-1.07)$ & 0.68 & $0.91(0.77-1.06)$ & 0.23 & 0.37 \\
\hline rs10183486 & $0.98(0.91-1.06)$ & 0.67 & $0.98(0.91-1.06)$ & 0.64 & $0.91(0.78-1.06)$ & 0.23 & 0.39 \\
\hline rs2307449 & $0.99(0.92-1.06)$ & 0.72 & $0.96(0.89-1.04)$ & 0.36 & $1.06(0.91-1.24)$ & 0.47 & 0.29 \\
\hline rs1635501 & $0.99(0.91-1.07)$ & 0.76 & $0.99(0.90-1.08)$ & 0.80 & $0.99(0.83-1.19)$ & 0.94 & 0.97 \\
\hline rs11668344 & 1.01 (0.94-1.08) & 0.81 & $1.00(0.92-1.08)$ & 0.92 & $1.05(0.89-1.22)$ & 0.58 & 0.59 \\
\hline rs1046089 & $0.99(0.92-1.07)$ & 0.89 & $1.02(0.94-1.10)$ & 0.67 & $0.90(0.77-1.05)$ & 0.19 & 0.17 \\
\hline rs365132 & $1.00(0.93-1.07)$ & 0.97 & $1.00(0.93-1.08)$ & 1.00 & $1.00(0.86-1.16)$ & 0.95 & 0.96 \\
\hline \multicolumn{8}{|l|}{ Breast cancer } \\
\hline rs11249433 & $1.20(1.12-1.29)$ & $4.8 \times 10^{-7}$ & $1.23(1.14-1.33)$ & $6.9 \times 10^{-8}$ & $1.06(0.91-1.23)$ & 0.45 & 0.081 \\
\hline rs3803662 & $1.20(1.12-1.30)$ & $1.8 \times 10^{-6}$ & $1.26(1.16-1.37)$ & $4.2 \times 10^{-8}$ & $0.98(0.82-1.16)$ & 0.78 & 0.008 \\
\hline rs2981582 & $1.18(1.10-1.27)$ & $8.2 \times 10^{-6}$ & $1.19(1.10-1.29)$ & $1.1 \times 10^{-5}$ & 1.01 (0.87-1.19) & 0.86 & 0.071 \\
\hline rs13281615 & $1.16(1.08-1.24)$ & $3.9 \times 10^{-5}$ & $1.16(1.07-1.25)$ & $2.1 \times 10^{-4}$ & $1.15(0.99-1.33)$ & 0.074 & 0.94 \\
\hline rs13387042 & $1.15(1.07-1.23)$ & $8.4 \times 10^{-5}$ & $1.17(1.08-1.26)$ & $6.3 \times 10^{-5}$ & $1.01(0.87-1.18)$ & 0.85 & 0.10 \\
\hline rs7716600 & $1.17(1.08-1.27)$ & $1.6 \times 10^{-4}$ & $1.18(1.08-1.29)$ & $2.9 \times 10^{-4}$ & $1.21(1.02-1.44)$ & 0.029 & 0.77 \\
\hline rs889312 & $1.10(1.02-1.19)$ & 0.012 & $1.10(1.01-1.20)$ & 0.026 & $1.18(1.00-1.39)$ & 0.046 & 0.45 \\
\hline rs999737 & $1.09(1.00-1.18)$ & 0.049 & $1.10(1.01-1.21)$ & 0.031 & $0.99(0.84-1.18)$ & 0.95 & 0.29 \\
\hline
\end{tabular}


Table 4 Association of candidate single-nucleotide polymorphism loci and risk of breast cancer by estrogen receptor status in the Nurses?'? Health Study and Women?'?s Genome Health Study (Continued)

\begin{tabular}{|c|c|c|c|c|c|c|c|}
\hline rs1045485 & $1.09(0.98-1.20)$ & 0.12 & $1.06(0.95-1.19)$ & 0.28 & $1.20(0.95-1.52)$ & 0.12 & 0.35 \\
\hline rs3817198 & $1.03(0.96-1.12)$ & 0.38 & $1.06(0.98-1.15)$ & 0.16 & $0.88(0.74-1.04)$ & 0.13 & 0.047 \\
\hline
\end{tabular}

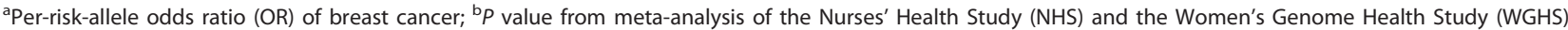
with additive genetic coding after adjustment for age and potential population stratification; ${ }^{c} P$ value from heterogeneity test across estrogen receptor-positive $\left(\mathrm{ER}^{+}\right)$and $\mathrm{ER}^{-}$tumors; ${ }^{d}$ total number of cases or controls in the NHS and the WGHS. Cl, confidence interval; SNP, single-nucleotide polymorphism.

menarche, rs17268785, was associated with a decreased risk of $\mathrm{ER}^{-}$tumors (per-allele $\mathrm{OR}=0.83 ; 95 \% \mathrm{CI}=0.68$ to 1.00 ) but an increased risk of $\mathrm{ER}^{+}$tumors (per-allele $\mathrm{OR}=1.07 ; 95 \% \mathrm{CI}=0.96$ to $1.19 ; P$ for heterogeneity $=$ 0.023). For the SNP that had a reported association with age at natural menopause and that was associated with overall breast cancer risk, we observed a stronger association with $\mathrm{ER}^{-}$tumors (per-allele $\mathrm{OR}=1.32$; 95\% $\mathrm{CI}=$ 1.09 to 1.61 ) than $\mathrm{ER}^{+}$tumors (per-allele $\mathrm{OR}=1.11$; $95 \% \mathrm{CI}=1.00$ to 1.24 ); however, the test for heterogeneity was not statistically significant ( $P$ for heterogeneity $=0.12$ ). When the count GRS for age at menarche or age at natural menopause was applied to $E R^{+}$and $E R^{-}$ breast cancer separately, the trend in the OR for $\mathrm{ER}^{+}$ tumors was very similar to that for overall breast cancer. The ER ${ }^{-}$tumor data suggested a somewhat different pattern, although the statistical power was limited for this subtype (Figure 2).

Of the 10 candidate SNPs with consistently reported associations with breast cancer risk, five SNPs (rs11249433, rs3803662, rs2981582, rs13387042, and rs999737) appeared to have a stronger association with $\mathrm{ER}^{+}$tumor than $\mathrm{ER}^{-}$tumors, and rs3803662 reached statistical significance $(P$ for heterogeneity $=0.008)$ with per-risk-allele ORs of 1.26 (95\% CI $=1.16$ to 1.37$)$ and 0.98 (95\% CI $=0.82$ to 1.16 ) for $\mathrm{ER}^{+}$and $\mathrm{ER}^{-}$tumors,

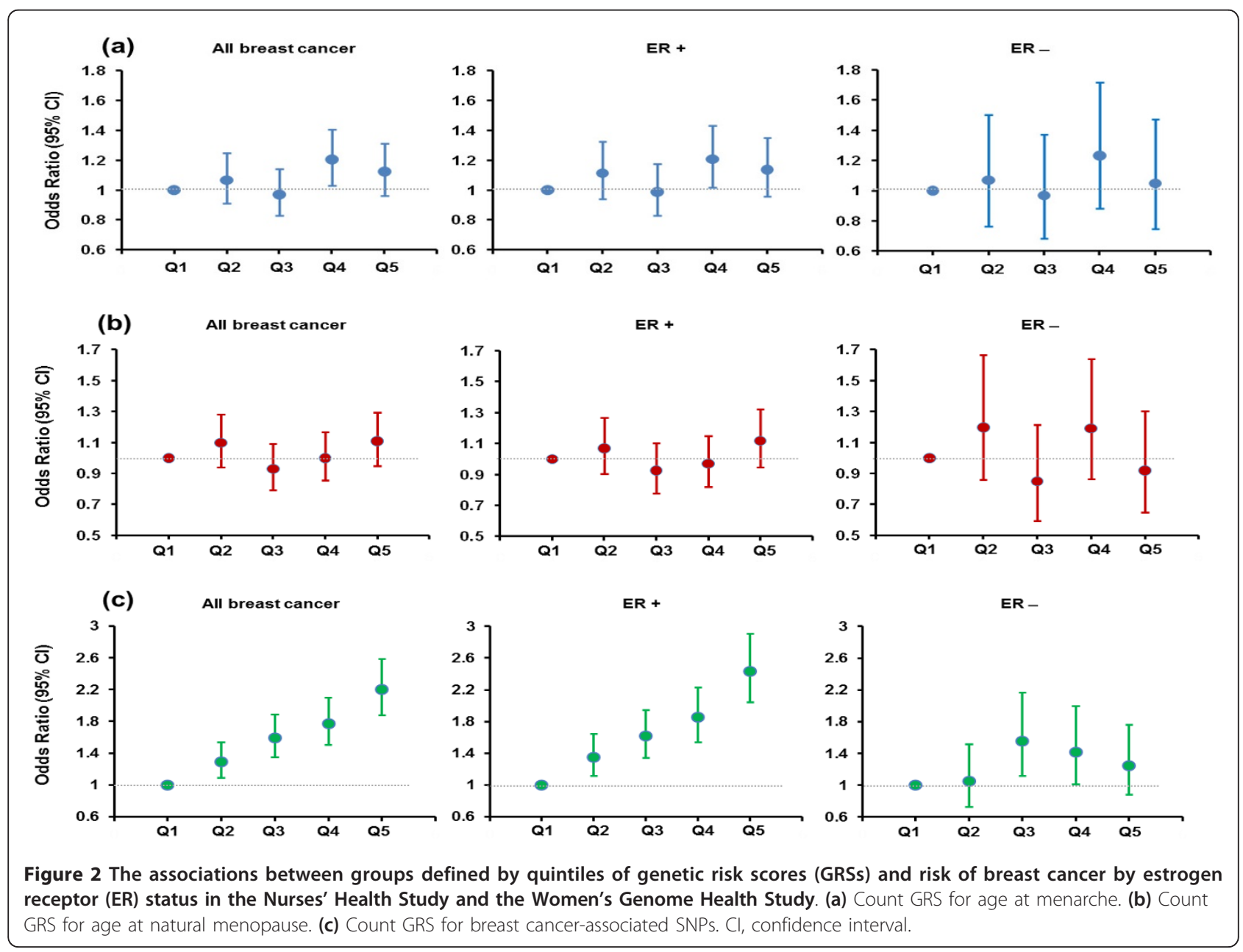


respectively (Table 4). Two breast cancer candidate SNPs, rs1045485 and rs3817198, did not show statistically significant associations with overall risk. However, rs1045485 appeared to have a stronger association with $E^{-}$tumors (per-allele $\mathrm{OR}=1.20 ; 95 \% \mathrm{CI}=0.95$ to 1.52) than $\mathrm{ER}^{+}$tumors (per-allele $\mathrm{OR}=1.06 ; 95 \% \mathrm{CI}=$ 0.95 to $1.19 ; P$ for heterogeneity $=0.35$ ), and $\mathrm{rs} 3817198$ was associated with a decreased risk of ER tumors (perallele $\mathrm{OR}=0.88 ; 95 \% \mathrm{CI}=0.74$ to 1.04 ) but an increased risk of $\mathrm{ER}^{+}$tumors (per-allele $\mathrm{OR}=1.06$; $95 \%$ $\mathrm{CI}=0.98$ to $1.15 ; P$ for heterogeneity $=0.047$ ).

In these analyses, we further confirmed statistically significant associations with breast cancer risk for 8 of the 10 candidate SNPs that were identified previously in published GWAS of breast cancer (most $P$ values were less than 0.001) (Table 2). We did not observe a statistically significant association for either LSP1-rs3817198 or CASP8-rs1045485 (both with $P$ values of 0.13 ) in our study, although the direction of the associations was consistent with that of previous reports [21,22]. We also calculated, as a positive control, a count GRS based on these 10 SNPs. We found that each score point increase, corresponding to one-risk-allele increase, was significantly associated with an OR of 1.13 (95\% CI $=1.11$ to 1.15) for breast cancer (Table 3). Compared with women in the lowest quintile, women in the highest quintile had an OR for breast cancer of 1.89 (95\% CI = 1.67 to 2.14). For this GRS, the trend in log odds was significantly steeper for $\mathrm{ER}^{+}$than for $\mathrm{ER}^{-}$tumors $(P$ for heterogeneity $<0.001$ ), and the OR across quintiles was no longer monotonic in $\mathrm{ER}^{-}$tumors (Figure 2).

\section{Discussion}

In this large meta-analysis of six population-based studies, we investigated whether 19 loci linked with age at menarche and 17 loci linked with age at natural menopause were associated with breast cancer risk among up to 3,683 breast cancer cases and 34,174 controls. We found that two SNPs with reported associations with age at menarche and one SNP with a reported association with age at natural menopause were significantly associated with breast cancer risk. However, no statistically significant associations were found for GRSs that combined all 19 or 17 loci associated with each trait, although the association for age-at-menarche GRS was marginally statistically significant. We confirmed most of the candidate loci for breast cancer which were identified in previous GWAS. Some of these associations appeared to differ by tumor subtypes defined by ER status.

In our analyses, most of the candidate SNPs, including the strongest GWAS hit for age at menarche or age at natural menopause, were not found to be associated with breast cancer risk. This is not necessarily surprising given that age at menarche and age at natural menopause are relatively weak risk factors [2,3], and all candidate SNPs collectively explain only a small portion of the variation of each trait $[10,11]$. However, two candidate SNPs for age at menarche, rs1079866 and rs7821178, and one candidate SNP for age at natural menopause, rs2517388, were found to be associated with breast cancer risk. These associations were not attenuated after we further adjusted for self-reported age at menarche and age at natural menopause, suggesting these three genetic loci were associated with breast cancer risk independently of their associations with age at menarche or age at natural menopause. It is possible that these genetic loci have pleiotropic effects on reproductive timing as well as other biological processes leading to breast cancer, and the observed associations might be due largely to other biological consequences of these risk variants that do not manifest themselves as changes in age at menarche or age at natural menopause. Alternatively, it is also possible that the relatively crude assignment of these reproductive events to a single chronological year is not sufficiently accurate to capture the biological effect of these processes on breast cancer risk and the genetic variants contribute independent information on the underlying biological risk. The three candidate SNPs also contributed to breast cancer risk independently of the known susceptibility loci for breast cancer, as further adjustment for breast cancer loci did not materially alter the results.

We found statistically significant evidence of association with breast cancer for eight of the 10 breast cancer susceptibility loci examined: FGFR2-rs2981582, TNRC9rs3803662, 1p-rs11249433, 5p-rs7716600, 2q35rs13387042, MAP3K1-rs889312, 8q24-rs13281615, and RAD51L1- rs999737. The direction and magnitude of these associations were consistent with those of previous reports [17,18,22-25]. We did not observe a statistically significant association for either LSP1-rs3817198 or CASP8-rs1045485. However, these two SNPs had relatively small reported effects that our study might not have been able to detect. When the 10 candidate SNPs were combined by using a polygenic risk score, the relative risk for women in the highest quintile was about twice that in the lowest quintile, and this is in accordance with other published results $[19,26]$. In this study, none of the 10 breast cancer susceptibility loci was significantly associated with age at menarche or age at natural menopause, and this is in line with a previous report [27].

Given that most of the candidate loci for age at menarche and age at natural menopause were not associated with breast cancer risk, it is not surprising that there were no statistically significant associations for the polygenetic risk scores that combined all candidate loci 
for each trait. To conduct a post hoc and exploratory analysis, we created a polygenetic risk score by including only the three candidate loci associated with either age at menarche or age at natural menopause and with breast cancer risk and found that each risk allele increment was associated with an approximately $17 \%$ increased risk for breast cancer. Women with four or more risk alleles had an approximately $60 \%$ increased risk for breast cancer in comparison with those with two risk alleles or less. When we further combined the three associated SNPs with the 10 breast cancer susceptibility loci to create a polygenetic risk score, each risk allele increment was associated with an approximately $18 \%$ increased risk for breast cancer. For women with 14 or more risk alleles (the highest quintile), the risk for breast cancer increased threefold in comparison with those with 10 or less (the lowest quintile). Because the former group constitutes approximately $20 \%$ of the study population, the GRS that combines the three candidate SNPs for age at menarche and age at natural menopause and the identified breast cancer susceptibility loci might be useful for identifying a subgroup of women with a high genetic risk for breast cancer. Further research is needed to confirm this finding.

It has been hypothesized that the risk of $\mathrm{ER}^{+}$breast cancer is positively associated with a woman's cumulative lifetime exposure to endogenous ovarian hormones [28]. A younger age at menarche [12,15,29] and an older age at menopause [30] have been observed to be more consistently associated with $\mathrm{ER}^{+}$than $\mathrm{ER}^{-}$tumors. In this report, we found that candidate loci for age at menarche and age at natural menopause may also be differentially associated with tumor subtypes defined by ER status. Of the three candidate loci that were found to be associated with overall breast cancer risk, rs1079866 was more strongly associated with $\mathrm{ER}^{+}$ tumors, rs7821178 was equally associated with both, whereas rs 2517388 was more strongly associated with $E R^{-}$tumors, although differences were not statistically significant. Importantly, two candidate loci for age at menarche, rs17188434 and rs17268785, had significantly different associations with $\mathrm{ER}^{+}$and $\mathrm{ER}^{-}$tumors. Whereas both SNPs were not significantly associated with overall and $\mathrm{ER}^{+}$breast cancer, the former showed a statistically significant positive association with $\mathrm{ER}^{-}$ tumors, whereas the latter showed a statistically significant inverse association with $\mathrm{ER}^{-}$tumor. These findings provide further support for the notion that $\mathrm{ER}^{+}$and $\mathrm{ER}^{-}$ tumors are the result of different etiologic pathways [31].

Although common genetic variants that influence the intermediate phenotypes or risk factors have been hypothesized to subsequently affect disease risk, few studies have assessed the association between these genetic variants and disease risk or, furthermore, whether these associations are mediated through the intermediate phenotypes. Chen and colleagues [32] investigated obesitylinked genetic variants in relation to breast cancer risk but found no statistically significant association. To our knowledge, ours is the first study to evaluate the associations of candidate loci for age at menarche and age at natural menopause with breast cancer risk. One of the strengths of our study is the relatively large combined sample size achieved through international collaboration. We had adequate statistical power $(80 \%)$ to detect an OR of 1.12 for SNPs with a minor allele frequency (MAF) of 0.10 and an OR of 1.09 for SNPs with an MAF of 0.20 . However, our analysis of $\mathrm{ER}^{+}$tumors was less adequately powered, as the ER status was not available for all cases, and the study had limited statistical power for $E^{-}$tumors. One limitation in our study is the multiple comparisons that could lead to false-positive results. Although none of the candidate SNPs with a reported association with age at menarche or age at natural menopause survived Bonferroni correction in the test of breast cancer association, this correction is considered to be overly conservative given that the candidates were chosen on the basis of promising hypotheses. Another potential limitation of our study comes from differences in the study population and designs and methods of collecting risk factors and genetic marker data across studies. However, the findings were generally consistent across studies, arguing for the robustness of our results. Finally, as our analyses were restricted to women of European ancestry, results from this study may not be generalizable to other ethnic groups.

\section{Conclusions}

In summary, in this large analysis of the association of several novel candidate loci for age at menarche and age at natural menopause with breast cancer risk, we observed that three loci - two for age at menarche and one for age at natural menopause - were significantly associated with breast cancer risk independently of their associations with each trait and independently of known breast cancer susceptibility loci. These associations may differ by tumor subtypes defined by ER status. A combination of all 19 loci associated with age at menarche or 17 loci associated with age at natural menopause did not appear to be helpful for identifying a high-risk subgroup for breast cancer.

\section{Additional material}

Additional file 1: Supplementary methods for study population. 
Additional file 2: Table S1: Information on the 46 candidate SNP loci identified in previous genome-wide association studies for age at menarche, age at natural menopause and breast cancer. Additional file 3: Table S2: Characteristics of non-genetic risk factors for breast cancer in each participating study.

\begin{abstract}
Abbreviations
ARIC: Atherosclerosis Risk in Communities Study; BMl: body mass index; Cl: confidence interval; ER: estrogen receptor; FHS: Framingham Heart Study; GRS: genetic risk score; GWAS: genome-wide association studies; MAF: minor allele frequency; NHS: Nurses' Health Study; OC: oral contraceptive; OR: odds ratio; RSI+II: Rotterdam Study I and II; SardiNIA: SardiNIA Breast Cancer Study; SNP: single-nucleotide polymorphism; WGHS: Women's Genome Health Study.
\end{abstract}

\section{Acknowledgements}

The NHS breast cancer GWAS scan was performed as part of the Cancer Genetic Markers of Susceptibility initiative of the National Cancer Institute $(\mathrm{NCl})$. We particularly acknowledge the contributions of Robert Hoover, Amy Hutchinson, Kevin Jacobs, and Gilles Thomas. The present research is supported by CA 40356 and U01-CA98233 from the NCl. We acknowledge the study participants in the NHS for their contribution in making this study possible. The WGHS is supported by HL 043851 and HL69757 from the National Heart, Lung, and Blood Institute (NHLBI) and CA 047988 from the $\mathrm{NCl}$, the Donald W Reynolds Foundation, and the Fondation Leduca, and collaborative scientific support and funding for genotyping were provided by Amgen (Thousand Oaks, CA, USA). We thank Joseph Miletich and Alex Parker and the technical staff at Amgen for their collaboration and scientific support in performing the genotyping for the WGHS. The SardiNIA Breast Cancer Study was funded by National Institutes of Health $(\mathrm{NIH})$ contract NO1-AG-1-2109 from the National Institute of Aging (NIA) to the SardiNIA team (principal investigator: Manuela Uda). We thank Grazia Palomba, Antonio Cossu, Francesco Tanda, Mario Budroni, and the other members of the Sardinian Translational Oncology Group for their contribution in collecting Sardinian patients with breast cancer. The generation and management of GWAS genotype data for the Rotterdam Study are supported by The Netherlands Organisation for Scientific Research (NWO) Investments (175.010.2005.011 and 911-03-012). This study is funded by the Research Institute for Diseases in the Elderly (RIDE2) (014-93-015) and The Netherlands Genomics Initiative/NWO project 050-060-810. We thank Pascal Arp, Mila Jhamai, Marijn Verkerk, Lizbeth Herrera, and Marjolein Peters for their help in creating the GWAS database and Karol Estrada and Maksim V Struchalin for their support in creation and analysis of imputed data. The Rotterdam Study is funded by the Erasmus Medical Center and Erasmus University, Rotterdam; the Netherlands Organisation for Health Research and Development (ZonMw); the Research Institute for Diseases in the Elderly (RIDE); the Ministry of Education, Culture and Science; the Ministry for Health, Welfare and Sports; the European Commission (DG XII); and the Municipality of Rotterdam. The authors are grateful to the study participants, the staff from the Rotterdam Study, and the participating general practitioners and pharmacists. The authors acknowledge the essential role of the Cohorts for Heart and Aging Research in Genome Epidemiology (CHARGE) Consortium in the development and support of this manuscript. CHARGE members consist of The Netherlands' Rotterdam Study, the FHS, the Cardiovascular Health Study, the NHLBI's ARIC study, and the NIA's Iceland Age, Gene/Environment Susceptibility (AGES) study. The FHS phenotype-genotype analyses were supported in part by the NIA (Genetics of Reproductive Life Period and Health Outcomes, R21AG032598; JMM, KLL). The FHS of the NHLBI of the $\mathrm{NIH}$ and Boston University School of Medicine was supported by NHLBI's FHS contract N01-HC-25195 and its contract with Affymetrix, Inc. (Santa Clara, CA, USA) for genotyping services (contract N02HL-6-4278). Analyses reflect intellectual input and resource development from the FHS investigators participating in the SNP Health Association Resource (SHARe) project. A portion of this research was conducted by using the Linux Cluster for Genetic Analysis (LinGA-II) funded by the Robert Dawson Evans Endowment of the Department of Medicine at Boston University School of Medicine and Boston Medical Center. The ARIC study is carried out as a collaborative study supported by NHLBI contracts N01-HC-
55015, N01-HC-55016, N01-HC-55018, N01-HC-55019, N01-HC-55020, N01-HC55021, N01-HC-55022, R01HL087641, R01HL59367, R01HL086694, and RC2 HL102419; National Human Genome Research Institute contract U01HG004402; and NIH contract HHSN268200625226C. The authors thank the staff and participants of the ARIC study for their important contributions. Infrastructure was partly supported by grant UL1RR025005, a component of the NIH, and NIH Roadmap for Medical Research.

\section{Author details}

${ }^{1}$ Department of Public Health, Indiana University School of Medicine, 980 West Walnut Street, R3-C241, Indianapolis, IN 46202, USA. Indiana University Melvin and Bren Simon Cancer Center, 535 Barnhill Drive, Indianapolis, IN 46202, USA. 3Division of Preventive Medicine, Brigham and Women's Hospital, Harvard Medical School, 900 Commonwealth Avenue East, Boston, MA 02215, USA. ${ }^{4}$ Division of Epidemiology and Community Health, School of Public Health, 1300 South Second Street, Suite 300, University of Minnesota, Minneapolis, MN 55454, USA. ${ }^{5}$ Framingham Heart Study, The National Heart Lung and Blood Institute, 73 Mount Wayte, Suite 2, Framingham, MA 01701, USA. ${ }^{6}$ Department of Epidemiology, Erasmus Medical Center, 3015 GE, Rotterdam, The Netherlands. ${ }^{7}$ Istituto di Ricerca Genetica e Biomedica, Consiglio Nazionale delle Ricerche, c/o Cittadella Universitaria di Monserrato, Monserrato, 09042 Cagliari, Italy. ${ }^{8}$ Department of Epidemiology, Gillings School of Global Public Health, University of North Carolina at Chapel Hill, 137 East Franklin Street, Suite 306, CB \#8050, Chapel Hill, NC 27599, USA. ${ }^{9}$ Department of Epidemiology, Harvard School of Public Health, 677 Huntington Avenue, Boston, MA 02115, USA. ${ }^{10}$ Channing Laboratory, Department of Medicine, Brigham and Women's Hospital, and Harvard medical School, 181 Longwood Avenue, Boston, MA 02115, USA. ${ }^{11}$ Department of Biostatistics, Boston University School of Public Health, 801 Massachusetts Avenue, 3rd floor, Boston, MA 02118, USA. ${ }^{12}$ Unit of Cancer Genetics, Istituto di Chimica Biomolecolare, Consiglio Nazionale delle Ricerche, Li Punti, 07100 Sassari, Italy. ${ }^{13}$ Department of Internal Medicine, Erasmus Medical Center, 3000 CA, Rotterdam, The Netherlands.

${ }^{14}$ Netherlands Consortium of Healthy Aging, Rotterdam, Rotterdam, The Netherlands. ${ }^{15}$ Division of Cancer Epidemiology and Genetics, National Cancer Institute, National Institutes of Health, Department of Health and Human Services, 6120 Executive Boulevard, Bethesda, MD 20892, USA. ${ }^{16}$ Sections of General Internal Medicine, Department of Medicine, Boston University School of Medicine, 720 East Concord Street, Boston, MA 02118, USA. ${ }^{17}$ Program in Medical and Population Genetics, Broad Institute of Harvard University and MIT, 301 Binney Street, Cambridge, MA 02142, USA.

\section{Authors' contributions}

$\mathrm{CH}$ performed the meta-analysis, wrote the manuscript, helped to conceive and design the experiments, helped to perform the primary statistical analyses in each study, shared responsibility for the interpretation of results and critical revision of the manuscript, and contributed to a critical revision of the manuscript for important intellectual content. DIC helped to conceive and design the experiments, helped to perform the primary statistical analyses in each study, shared responsibility for the interpretation of results and critical revision of the manuscript, and contributed to a critical revision of the manuscript for important intellectual content. DJH helped to conceive and design the experiments, contributed to a critical revision of the manuscript for important intellectual content, and participated in the original design, subject recruitment, acquisition of data, biospecimen collection for the studies, and the genotyping and quality control of genotype and other data. JD, SJH, RR, and SS helped to perform the primary statistical analyses in each study and shared responsibility for the interpretation of results and critical revision of the manuscript. SJC, LC, EWD, JMM, PMR, and BHS contributed to a critical revision of the manuscript for important intellectual content and participated in the original design, subject recruitment, acquisition of data, biospecimen collection for the studies, and the genotyping and quality control of genotype and other data. JEB, LF-R, NF, SEH, AH, KLL, GP, EP, FR, LMR, GLS, LS, and AGU participated in the original design, subject recruitment, acquisition of data, biospecimen collection for the studies, and the genotyping and quality control of genotype and other data. All authors read and approved the final manuscript.

\section{Competing interests}

The authors declare that they have no competing interests. 
Received: 10 November 2011 Revised: 31 January 2012

Accepted: 20 March 2012 Published: 20 March 2012

\section{References}

1. Kelsey $\mathrm{JL}$, Horn-Ross PL: Breast cancer: magnitude of the problem and descriptive epidemiology. Epidemiol Rev 1993, 15:7-16.

2. Hsieh CC, Trichopoulos D, Katsouyanni K, Yuasa S: Age at menarche, age at menopause, height and obesity as risk factors for breast cancer: associations and interactions in an international case-control study. Int J Cancer 1990, 46:796-800.

3. Breast cancer and hormone replacement therapy: collaborative reanalysis of data from 51 epidemiological studies of 52,705 women with breast cancer and 108,411 women without breast cancer. Collaborative Group on Hormonal Factors in Breast Cancer. Lancet 1997, 350:1047-1059.

4. Pike MC, Spicer DV, Dahmoush L, Press MF: Estrogens, progestogens, normal breast cell proliferation, and breast cancer risk. Epidemiol Rev 1993, 15:17-35.

5. He C, Kraft P, Chen C, Buring JE, Pare G, Hankinson SE, Chanock SJ, Ridker PM, Hunter DJ, Chasman DI: Genome-wide association studies identify loci associated with age at menarche and age at natural menopause. Nat Genet 2009, 41:724-728.

6. Ong KK, Elks CE, Li S, Zhao JH, Luan J, Andersen LB, Bingham SA, Brage $S$, Smith GD, Ekelund U, Gillson CJ, Glaser B, Golding J, Hardy R, Khaw KT, Kuh D, Luben R, Marcus M, McGeehin MA, Ness AR, Northstone K, Ring SM, Rubin C, Sims MA, Song K, Strachan DP, Vollenweider P, Waeber G, Waterworth DM, Wong A, et al: Genetic variation in LIN28B is associated with the timing of puberty. Nat Genet 2009, 41:729-733.

7. Perry JR, Stolk L, Franceschini N, Lunetta KL, Zhai G, McArdle PF, Smith AV, Aspelund T, Bandinelli S, Boerwinkle E, Cherkas L, Eiriksdottir G, Estrada K, Ferrucci L, Folsom AR, Garcia M, Gudnason V, Hofman A, Karasik D, Kiel DP, Launer $L$, van Meurs J, Nalls MA, Rivadeneira F, Shuldiner AR, Singleton A, Soranzo N, Tanaka T, Visser JA, Weedon MN, et al: Meta-analysis of genome-wide association data identifies two loci influencing age at menarche. Nat Genet 2009, 41:648-650.

8. Sulem P, Gudbjartsson DF, Rafnar T, Holm H, Olafsdottir EJ, Olafsdottir GH, Jonsson T, Alexandersen P, Feenstra B, Boyd HA, Aben KK, Verbeek AL, Roeleveld N, Jonasdottir A, Styrkarsdottir U, Steinthorsdottir V, Karason A, Stacey SN, Gudmundsson J, Jakobsdottir M, Thorleifsson G, Hardarson G, Gulcher J, Kong A, Kiemeney LA, Melbye M, Christiansen C, Tryggvadottir L, Thorsteinsdottir U, Stefansson K: Genome-wide association study identifies sequence variants on $6 \mathrm{q} 21$ associated with age at menarche. Nat Genet 2009, 41:734-738.

9. Stolk L, Zhai G, van Meurs JB, Verbiest MM, Visser JA, Estrada K, Rivadeneira F, Williams FM, Cherkas L, Deloukas P, Soranzo N, de Keyzer JJ, Pop VJ, Lips P, Lebrun CE, van der Schouw YT, Grobbee DE, Witteman J, Hofman A, Pols HA, Laven JS, Spector TD, Uitterlinden AG: Loci at chromosomes 13,19 and 20 influence age at natural menopause. Nat Genet 2009, 41:645-647.

10. Elks CE, Perry JR, Sulem P, Chasman DI, Franceschini N, He C, Lunetta KL, Visser JA, Byrne EM, Cousminer DL, Gudbjartsson DF, Esko T, Feenstra B, Hottenga JJ, Koller DL, Kutalik Z, Lin P, Mangino M, Marongiu M, McArdle PF, Smith AV, Stolk L, van Wingerden SH, Zhao JH, Albrecht E, Corre T, Ingelsson E, Hayward C, Magnusson PK, Smith EN, et al: Thirty new loci for age at menarche identified by a meta-analysis of genome-wide association studies. Nat Genet 2010, 42:1077-1085.

11. Stolk L, Perry JR, Chasman DI, He C, Mangino M, Sulem P, Barbalic M, Broer L, Byrne EM, Ernst F, Esko T, Franceschini N, Gudbjartsson DF, Hottenga JJ, Kraft P, McArdle PF, Porcu E, Shin SY, Smith AV, van Wingerden S, Zhai G, Zhuang W, Albrecht E, Alizadeh BZ, Aspelund T, Bandinelli S, Lauc LB, Beckmann JS, Boban M, Boerwinkle E, et al: Metaanalyses identify 13 loci associated with age at menopause and highlight DNA repair and immune pathways. Nat Genet 2012, 44:260-268.

12. Althuis MD, Fergenbaum JH, Garcia-Closas M, Brinton LA, Madigan MP, Sherman ME: Etiology of hormone receptor-defined breast cancer: a systematic review of the literature. Cancer Epidemiol Biomarkers Prev 2004, 13:1558-1568

13. Kotsopoulos J, Chen WY, Gates MA, Tworoger SS, Hankinson SE, Rosner BA: Risk factors for ductal and lobular breast cancer: results from the nurses' health study. Breast Cancer Res 2010, 12:R106.
14. Li Cl, Malone KE, Daling JR, Potter JD, Bernstein L, Marchbanks PA, Strom BL, Simon MS, Press MF, Ursin G, Burkman RT, Folger SG, Norman S, McDonald JA, Spirtas R: Timing of menarche and first full-term birth in relation to breast cancer risk. Am J Epidemiol 2008, 167:230-239.

15. Ma H, Bernstein L, Pike MC, Ursin G: Reproductive factors and breast cancer risk according to joint estrogen and progesterone receptor status: a meta-analysis of epidemiological studies. Breast Cancer Res 2006, 8:R43.

16. Reeves GK, Pirie K, Green J, Bull D, Beral V: Reproductive factors and specific histological types of breast cancer: prospective study and metaanalysis. Br J Cancer 2009, 100:538-544.

17. Thomas $G$, Jacobs KB, Kraft P, Yeager M, Wacholder S, Cox DG, Hankinson SE, Hutchinson A, Wang Z, Yu K, Chatterjee N, Garcia-Closas M, Gonzalez-Bosquet J, Prokunina-Olsson L, Orr N, Willett WC, Colditz GA, Ziegler RG, Berg CD, Buys SS, McCarty CA, Feigelson HS, Calle EE, Thun MJ, Diver R, Prentice R, Jackson R, Kooperberg C, Chlebowski R, Lissowska J, et al: A multistage genome-wide association study in breast cancer identifies two new risk alleles at 1p11.2 and 14q24.1 (RAD51L1). Nat Genet 2009, 41:579-584.

18. Pharoah PD, Antoniou AC, Easton DF, Ponder BA: Polygenes, risk prediction, and targeted prevention of breast cancer. N Engl J Med 2008, 358:2796-2803.

19. Wacholder S, Hartge P, Prentice R, Garcia-Closas M, Feigelson HS, Diver WR, Thun MJ, Cox DG, Hankinson SE, Kraft P, Rosner B, Berg CD, Brinton LA, Lissowska J, Sherman ME, Chlebowski R, Kooperberg C, Jackson RD, Buckman DW, Hui P, Pfeiffer R, Jacobs KB, Thomas GD, Hoover RN, Gail MH, Chanock SJ, Hunter DJ: Performance of common genetic variants in breast-cancer risk models. N Engl J Med 2010, 362:986-993.

20. Higgins JP, Thompson SG: Quantifying heterogeneity in a meta-analysis. Stat Med 2002, 21:1539-1558.

21. Cox A, Dunning AM, Garcia-Closas M, Balasubramanian S, Reed MW, Pooley KA, Scollen S, Baynes C, Ponder BA, Chanock S, Lissowska J, Brinton L, Peplonska B, Southey MC, Hopper JL, McCredie MR, Giles GG, Fletcher O, Johnson N, dos Santos Silva I, Gibson L, Bojesen SE, Nordestgaard BG, Axelsson CK, Torres D, Hamann U, Justenhoven C, Brauch $\mathrm{H}$, Chang-Claude J, Kropp S, et al: A common coding variant in CASP8 is associated with breast cancer risk. Nat Genet 2007, 39:352-358.

22. Easton DF, Pooley KA, Dunning AM, Pharoah PD, Thompson D, Ballinger DG, Struewing JP, Morrison J, Field H, Luben R, Wareham N, Ahmed S, Healey CS, Bowman R, Meyer KB, Haiman CA, Kolonel LK, Henderson BE, Le Marchand L, Brennan P, Sangrajrang S, Gaborieau V Odefrey F, Shen CY, Wu PE, Wang HC, Eccles D, Evans DG, Peto J, Fletcher $\mathrm{O}$, et al: Genome-wide association study identifies novel breast cancer susceptibility loci. Nature 2007, 447:1087-1093.

23. Stacey SN, Manolescu A, Sulem P, Rafnar T, Gudmundsson J, Gudjonsson SA, Masson G, Jakobsdottir M, Thorlacius S, Helgason A, Aben KK, Strobbe LJ, Albers-Akkers MT, Swinkels DW, Henderson BE, Kolonel LN, Le Marchand L, Millastre E, Andres R, Godino J, Garcia-Prats MD, Polo E, Tres A, Mouy M, Saemundsdottir J, Backman VM, Gudmundsson L, Kristjansson K, Bergthorsson JT, Kostic J, et al: Common variants on chromosomes $2 \mathrm{q} 35$ and $16 \mathrm{q} 12$ confer susceptibility to estrogen receptor-positive breast cancer. Nat Genet 2007, 39:865-869.

24. Hunter DJ, Kraft P, Jacobs KB, Cox DG, Yeager M, Hankinson SE, Wacholder S, Wang Z, Welch R, Hutchinson A, Wang J, Yu K, Chatterjee N, Orr N, Willett WC, Colditz GA, Ziegler RG, Berg CD, Buys SS, McCarty CA, Feigelson HS, Calle EE, Thun MJ, Hayes RB, Tucker M, Gerhard DS, Fraumeni JF Jr, Hoover RN, Thomas G, Chanock SJ: A genome-wide association study identifies alleles in FGFR2 associated with risk of sporadic postmenopausal breast cancer. Nat Genet 2007, 39:870-874.

25. Stacey SN, Manolescu A, Sulem P, Thorlacius S, Gudjonsson SA, Jonsson GF, Jakobsdottir M, Bergthorsson JT, Gudmundsson J, Aben KK, Strobbe LJ, Swinkels DW, van Engelenburg KC, Henderson BE, Kolonel LN, Le Marchand L, Millastre E, Andres R, Saez B, Lambea J, Godino J, Polo E, Tres A, Picelli S, Rantala J, Margolin S, Jonsson T, Sigurdsson H, Jonsdottir T, Hrafnkelsson J, et al: Common variants on chromosome 5 p12 confer susceptibility to estrogen receptor-positive breast cancer. Nat Genet 2008, 40:703-706.

26. Reeves GK, Travis RC, Green J, Bull D, Tipper S, Baker K, Beral V, Peto R, Bell J, Zelenika D, Lathrop M: Incidence of breast cancer and its subtypes in relation to individual and multiple low-penetrance genetic susceptibility loci. JAMA 2010, 304:426-434. 
27. Travis RC, Reeves GK, Green J, Bull D, Tipper SJ, Baker K, Beral V, Peto R, Bell J, Zelenika D, Lathrop M: Gene-environment interactions in 7610 women with breast cancer: prospective evidence from the Million Women Study. Lancet 2010, 375:2143-2151.

28. Clavel-Chapelon F: Differential effects of reproductive factors on the risk of pre- and postmenopausal breast cancer. Results from a large cohort of French women. Br J Cancer 2002, 86:723-727.

29. Yang XR, Chang-Claude J, Goode EL, Couch FJ, Nevanlinna H, Milne RL, Gaudet M, Schmidt MK, Broeks A, Cox A, Fasching PA, Hein R, Spurdle AB, Blows F, Driver K, Flesch-Janys D, Heinz J, Sinn P, Vrieling A, Heikkinen T, Aittomaki K, Heikkila P, Blomqvist C, Lissowska J, Peplonska B, Chanock S, Figueroa J, Brinton L, Hall P, Czene K, et al: Associations of breast cancer risk factors with tumor subtypes: a pooled analysis from the Breast Cancer Association Consortium studies. J Natl Cancer Inst 2011, 103:250-263.

30. Phipps Al, Chlebowski RT, Prentice R, McTiernan A, Wactawski-Wende J, Kuller LH, Adams-Campbell LL, Lane D, Stefanick ML, Vitolins M, Kabat GC, Rohan TE, Li Cl: Reproductive history and oral contraceptive use in relation to risk of triple-negative breast cancer. J Natl Cancer Inst 2011, 103:470-477.

31. Allred DC, Brown P, Medina D: The origins of estrogen receptor alphapositive and estrogen receptor alpha-negative human breast cancer. Breast Cancer Res 2004, 6:240-245.

32. Chen F, Wilkens LR, Monroe KR, Stram DO, Kolonel LN, Henderson BE, Le Marchand L, Haiman CA: No association of risk variants for diabetes and obesity with breast cancer: the Multiethnic Cohort and PAGE studies. Cancer Epidemiol Biomarkers Prev 2011, 20:1039-1042.

doi:10.1186/bcr3155

Cite this article as: He et al:: Reproductive aging-associated common genetic variants and the risk of breast cancer. Breast Cancer Research 2012 14:R54.

\section{Submit your next manuscript to BioMed Central and take full advantage of:}

- Convenient online submission

- Thorough peer review

- No space constraints or color figure charges

- Immediate publication on acceptance

- Inclusion in PubMed, CAS, Scopus and Google Scholar

- Research which is freely available for redistribution

Submit your manuscript at www.biomedcentral.com/submit 